\title{
The Rapidity Distributions and the Thermalization Induced Transverse Momentum Distributions in Au-Au Collisions at RHIC Energies
}

\author{
Zhi-Jin Jiang, Jia-Qi Hui, and Yu Zhang \\ College of Science, University of Shanghai for Science and Technology, Shanghai 200093, China \\ Correspondence should be addressed to Zhi-Jin Jiang; jzj265@163.com
}

Received 23 January 2017; Revised 28 April 2017; Accepted 9 May 2017; Published 4 July 2017

Academic Editor: Sally Seidel

Copyright (c) 2017 Zhi-Jin Jiang et al. This is an open access article distributed under the Creative Commons Attribution License, which permits unrestricted use, distribution, and reproduction in any medium, provided the original work is properly cited. The publication of this article was funded by SCOAP ${ }^{3}$.

It is widely believed that the quark-gluon plasma (QGP) might be formed in the current heavy ion collisions. It is also widely recognized that the relativistic hydrodynamics is one of the best tools for describing the process of expansion and hadronization of QGP. In this paper, by taking into account the effects of thermalization, a hydrodynamic model including phase transition from QGP state to hadronic state is used to analyze the rapidity and transverse momentum distributions of identified charged particles produced in heavy ion collisions. A comparison is made between the theoretical results and experimental data. The theoretical model gives a good description of the corresponding measurements made in Au-Au collisions at RHIC energies.

\section{Introduction}

The primary goal of experimental program performed at Relativistic Heavy Ion Collider (RHIC) at Brookhaven National Laboratory (BNL) and at Large Hadron Collider (LHC) at CERN is to create a hot and dense matter consisting of partonic degrees of freedom, usually called the quark-gluon plasma (QGP), which is believed to have filled in the early universe several microseconds after the big bang. The calculations of Lattice Quantum Chromodynamics (LQCD) have predicted [1] that such matter may exist in the environment with critical temperature of about $T_{\mathrm{c}}=180 \mathrm{MeV}$ or energy density $\varepsilon_{\mathrm{c}}=2 \mathrm{GeV} / \mathrm{fm}^{3}$. By means of the Bjorken estimation [2] and the measurements of PHENIX Collaboration at RHIC, the spatial energy density in central Au-Au collisions at $\sqrt{s_{\mathrm{NN}}}=200$ and $130 \mathrm{GeV}$ is evaluated to be much higher than $\varepsilon_{c}$ [3]. Further studies have shown that QGP might be indeed generated in these collisions [4-7]. In fact, it has long been argued that QGP might even have come into being in $p-p(\bar{p})$ collisions at the energies of Intersecting Storage Rings (ISR) and Super Proton Synchrotron (SPS) at CERN [8-12].
In the past decade, a number of bulk observables about charged particles, such as the Fourier harmonic coefficients $v_{n}$ of azimuth-angle distributions $[13,14]$, rapidity or pseudorapidity distributions [15-18], and transverse momentum distributions [19-24], have experienced a series of extensive investigations in heavy ion collisions at both RHIC and LHC energies. These investigations have provided us with a compelling evidence that the matter created in heavy ion collisions exhibits a clear collective behavior, expanding nearly like a perfect fluid with very low viscosity. This sets up the prominent position of relativistic hydrodynamics in analyzing the properties of bulk observables in heavy ion collisions [25-46].

Apart from collective movement, the quanta of produced matter also have the components of thermal motion stemmed from the thermalization of fluid. The evolution of produced matter is then the superposition of these two parts. To clarify the role of thermalization in the expansions of the produced matter is the major subject of this paper. To this end, we may as usual in analytical treatment ignore the collective flow in the transverse directions. The transverse movement 
of the produced matter is therefore only induced by the thermalization.

The collective movement of produced matter in the longitudinal direction can be solved analytically. There are a few schemes in dealing with such exact calculations. In this paper, the hydrodynamic model proposed by Suzuki is employed [25]. Besides the analyticity, the other typical feature of this model is that it, like some other models [26-29], incorporates the effects of phase transition into solutions. This coincides with the current experimental observations as mentioned above. Hence, the employed model might be more in line with the realistic situations. In addition, the model is related to the initial temperature of QGP, the sound speed in both partonic and hadronic media, the baryochemical potential, and the critical temperature of phase transition. This work may therefore help us understand various transport coefficients of expanding system.

In Section 2, a brief introduction is given to the theoretical model [25], presenting its analytical formulations. The solutions are then used in Section 3 to formulate the invariant multiplicity distributions of charged particles produced in heavy ion collisions which are in turn compared with the experimental measurements carried out by BRAHMS and PHENIX Collaboration in Au-Au collisions at RHIC energies of $\sqrt{s_{\mathrm{NN}}}=200$ and $130 \mathrm{GeV}$ [16-20]. Section 4 is about conclusions.

\section{A Brief Introduction to the Model}

The main content of the theoretical model [25] is as follows.

(1) In the process of expansions, the energy and momentum of fluid are conserved. Hence, the movement of fluid follows the continuity equation

$$
\frac{\partial T^{\mu \nu}}{\partial x^{\nu}}=0, \quad \mu, \nu=0,1
$$

where $x^{\nu}=\left(x^{0}, x^{1}\right)=(t, z), t$ is the time, and $z$ is the longitudinal coordinate along beam direction. $T^{\mu v}$ is the energy-momentum tensor, which, for a perfect fluid, takes the form

$$
T^{\mu \nu}=(\varepsilon+p) u^{\mu} u^{\nu}-p g^{\mu \nu},
$$

where $g^{\mu \nu}=g_{\mu \nu}=\operatorname{diag}(1,-1)$ is the metric tensor. $u^{\mu}=\left(u^{0}\right.$, $\left.u^{1}\right)=\left(\cosh y_{\mathrm{L}}, \sinh y_{\mathrm{L}}\right)$ is the 4-velocity of fluid with rapidity $y_{\mathrm{L}} \cdot \varepsilon$ and $p$ in (2) are the energy density and pressure of fluid, related by the equation of state

$$
\frac{\mathrm{d} p}{\mathrm{~d} \varepsilon}=\frac{s \mathrm{~d} T}{T \mathrm{~d} s}=c_{\mathrm{s}}^{2}
$$

where $T, s$, and $c_{\mathrm{s}}$ are the temperature, entropy density, and the sound speed of fluid, respectively.
(2) In order to solve (1), Khalatnikov potential $\chi$ is introduced which makes the coordinate base of $(t, z)$ transform to that of $\left(\omega, y_{\mathrm{L}}\right)$ via relations

$$
\begin{aligned}
& t=\frac{e^{\omega}}{T_{0}}\left(\frac{\partial \chi}{\partial \omega} \cosh y_{\mathrm{L}}+\frac{\partial \chi}{\partial y_{\mathrm{L}}} \sinh y_{\mathrm{L}}\right), \\
& z=\frac{e^{\omega}}{T_{0}}\left(\frac{\partial \chi}{\partial \omega} \sinh y_{\mathrm{L}}+\frac{\partial \chi}{\partial y_{\mathrm{L}}} \cosh y_{\mathrm{L}}\right),
\end{aligned}
$$

where $T_{0}$ is the initial temperature of fluid and $\omega=\ln \left(T_{0} / T\right)$. Equation (1) is translated to the so-called telegraphy equation

$$
\frac{\partial^{2} \chi}{\partial \omega^{2}}-2 \beta \frac{\partial \chi}{\partial \omega}-\frac{1}{c_{\mathrm{s}}^{2}} \frac{\partial^{2} \chi}{\partial y_{\mathrm{L}}^{2}}=0, \quad \beta=\frac{1-c_{\mathrm{s}}^{2}}{2 c_{\mathrm{s}}^{2}} .
$$

(3) Along with the expansions of matter created in collisions, its temperature becomes lower and lower. As the temperature drops from initial $T_{0}$ to critical $T_{c}$, phase transition occurs. The matter transforms from QGP state to hadronic state. The produced hadrons are initially in the violent and frequent collisions. The major part of these collisions is inelastic. Hence, the abundance of an identified hadron is in changing. Furthermore, the mean free paths of these primary hadrons are very short. The movement of them is still like that of a fluid meeting (5) with only difference being the value of $c_{\mathrm{s}}$. In QGP, $c_{\mathrm{s}}=c_{0}=1 / \sqrt{3}$, which is the sound speed of a massless perfect fluid, being the maximum of $c_{\mathrm{s}}$. In the hadronic state, $c_{\mathrm{s}}=c_{\mathrm{h}}<c_{0}$. At the point of phase transition; that is, as $T=T_{\mathrm{c}}, c_{\mathrm{s}}$ is discontinuous.

(4) The solutions of (5) for the sectors of QGP and hadrons are, respectively [25],

$$
\begin{aligned}
& \chi_{0}\left(\omega, y_{\mathrm{L}}\right)=\frac{Q_{0} c_{0}}{2} e^{\beta_{0} \omega} I_{0}\left(\beta_{0} c_{0} \sqrt{y_{0}^{2}(\omega)-y_{\mathrm{L}}^{2}}\right), \\
& \chi_{\mathrm{h}}\left(\omega, y_{\mathrm{L}}\right)=\frac{Q_{0} c_{0}}{2} B(\omega) I_{0}\left[\lambda\left(\omega, y_{\mathrm{L}}\right)\right],
\end{aligned}
$$

where $Q_{0}$ is a constant determined by tuning the theoretical results to experimental data. $I_{0}$ is the 0th-order modified Bessel function of the first kind, and

$$
\begin{aligned}
\beta_{0} & =\frac{1-c_{0}^{2}}{2 c_{0}^{2}}=1, \\
y_{0}(\omega) & =\frac{\omega}{c_{0}}, \\
B(\omega) & =e^{\beta_{\mathrm{h}}\left(\omega-\omega_{\mathrm{c}}\right)+\beta_{0} \omega_{\mathrm{c}},} \\
\lambda\left(\omega, y_{\mathrm{L}}\right) & =\beta_{\mathrm{h}} c_{\mathrm{h}} \sqrt{y_{\mathrm{h}}^{2}(\omega)-y_{\mathrm{L}}^{2}}, \\
\beta_{\mathrm{h}} & =\frac{1-c_{\mathrm{h}}^{2}}{2 c_{\mathrm{h}}^{2}}, \\
\omega_{\mathrm{c}} & =\ln \left(\frac{T_{0}}{T_{\mathrm{c}}}\right), \\
y_{\mathrm{h}}(\omega) & =\frac{\omega-\omega_{\mathrm{c}}}{c_{\mathrm{h}}}+\frac{\omega_{\mathrm{c}}}{c_{0}} .
\end{aligned}
$$


It is evident that if $c_{\mathrm{h}}=c_{0}$, then $\beta_{\mathrm{h}}=\beta_{0}, B(\omega)=e^{\beta_{0} \omega}, y_{\mathrm{h}}=y_{0}$, and thus $\chi_{\mathrm{h}}=\chi_{0}$. At the point of phase transition, $T=T_{\mathrm{c}}$, $\omega=\omega_{\mathrm{c}}, B(\omega)=e^{\beta_{0} \omega_{\mathrm{c}}}$, and $y_{\mathrm{h}}=y_{0}=\omega_{\mathrm{c}} / c_{0}$. Then

$$
\begin{aligned}
& \left.\chi_{0}\left(\omega, y_{\mathrm{L}}\right)\right|_{T=T_{\mathrm{c}}}=\frac{\mathrm{Q}_{0} c_{0}}{2} e^{\beta_{0} \omega_{\mathrm{c}}} I_{0}\left(\beta_{0} \sqrt{\omega_{\mathrm{c}}^{2}-c_{0}^{2} y_{\mathrm{L}}^{2}}\right), \\
& \left.\chi_{\mathrm{h}}\left(\omega, y_{\mathrm{L}}\right)\right|_{T=T_{\mathrm{c}}}=\frac{Q_{0} c_{0}}{2} e^{\beta_{0} \omega_{\mathrm{c}}} I_{0}\left(\frac{\beta_{\mathrm{h}} c_{\mathrm{h}}}{c_{0}} \sqrt{\omega_{\mathrm{c}}^{2}-c_{0}^{2} y_{\mathrm{L}}^{2}}\right),
\end{aligned}
$$

$\left.\chi_{\mathrm{h}}\left(\omega, y_{\mathrm{L}}\right)\right|_{T=T_{\mathrm{c}}} \neq\left.\chi_{0}\left(\omega, y_{\mathrm{L}}\right)\right|_{T=T_{c}}$. That is, the potential $\chi$ is discontinuous at point of $T=T_{c}$.

\section{The Rapidity Distributions and the Thermalization Induced Transverse Momentum Distributions of Identified Charged Particles}

With the expansions of hadronic matter, its temperature continues becoming lower. According to the prescription of Cooper-Frye [31], as the temperature drops to the socalled chemical freeze-out temperature $T_{\mathrm{FO}}$, the inelastic collisions among hadrons cease. The abundance of an identified hadron maintains unchanged becoming the measured results in experiments. The invariant multiplicity distributions of charged particles are given by $[25,31,32]$

$$
\begin{aligned}
& \frac{\mathrm{d}^{2} N}{2 \pi p_{\mathrm{T}} \mathrm{d} y \mathrm{~d} p_{\mathrm{T}}} \\
& =\left.\frac{\mathrm{Q}_{0} c_{0}}{2} \frac{A(b)}{(2 \pi)^{3}} \int\left(\cosh y \frac{\mathrm{d} z}{\mathrm{~d} y_{\mathrm{L}}}-\sinh y \frac{\mathrm{d} t}{\mathrm{~d} y_{\mathrm{L}}}\right)\right|_{T=T_{\mathrm{FO}}} \\
& \quad \times \frac{m_{\mathrm{T}} \cosh \left(y-y_{\mathrm{L}}\right)}{\exp \left\{\left[m_{\mathrm{T}} \cosh \left(y-y_{\mathrm{L}}\right)-\mu_{\mathrm{B}}\right] / T_{\mathrm{FO}}\right\}+\delta} \mathrm{d} y_{\mathrm{L}},
\end{aligned}
$$

where $A(b)$ is the area of overlap region of collisions, being the function of impact parameter $b$ or centrality cuts. $m_{\mathrm{T}}=$ $\sqrt{m^{2}+p_{\mathrm{T}}^{2}}$ is the transverse mass of produced charged particle with rest mass $m . \mu_{\mathrm{B}}$ in (9) is the baryochemical potential. For Fermi charged particles, $\delta=1$ in the denominator of (9), and for Bosons, $\delta=-1$. That is, Fermi and Bose charged particles follow the Fermi-Dirac and Bose-Einstein distributions, respectively. The meaning of (9) is evident. The part of integrand in the round brackets is proportional to the rapidity density of fluid resulting from the collective movement along longitudinal direction [31]. The rest part is the energy of the charged particles in the state with temperature $T_{\mathrm{FO}}$ and transverse mass $m_{\mathrm{T}}$ resulting from the thermalization of fluid.

From (4) and (8), it can be shown that

$$
\begin{aligned}
& \left.\left(\cosh y \frac{\mathrm{d} z}{\mathrm{~d} y_{\mathrm{L}}}-\sinh y \frac{\mathrm{d} t}{\mathrm{~d} y_{\mathrm{L}}}\right)\right|_{T=T_{\mathrm{FO}}}=\frac{1}{T_{\mathrm{FO}}}\left(\beta_{\mathrm{h}} c_{\mathrm{h}}\right)^{2} \\
& \cdot B\left(\omega_{\mathrm{FO}}\right)\left[S\left(\omega_{\mathrm{FO}}, y_{\mathrm{L}}\right) \sinh \left(y-y_{\mathrm{L}}\right)\right. \\
& \left.+C\left(\omega_{\mathrm{FO}}, y_{\mathrm{L}}\right) \cosh \left(y-y_{\mathrm{L}}\right)\right]
\end{aligned}
$$

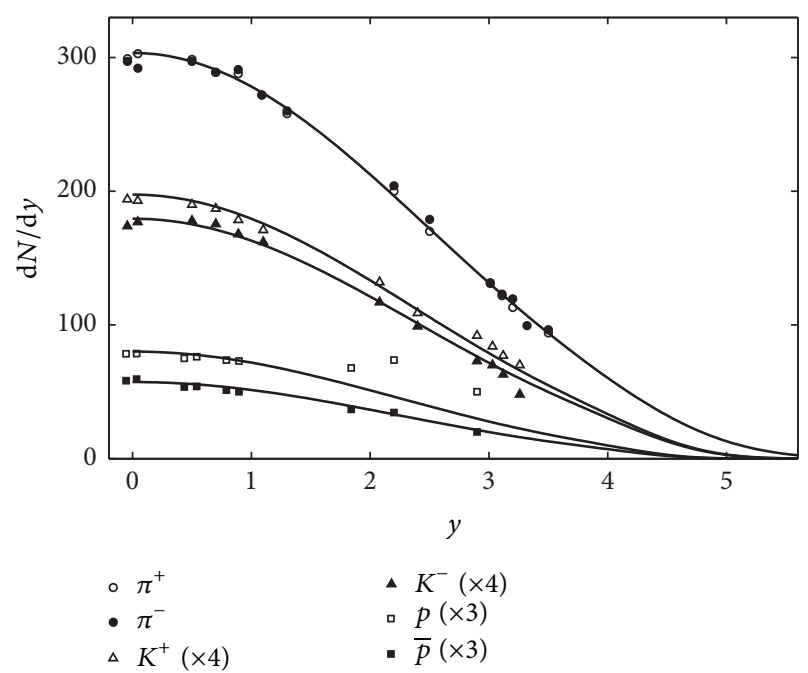

Figure 1: The rapidity distributions of $\pi^{ \pm}, K^{ \pm}$, and $p(\bar{p})$ in central $\mathrm{Au}-\mathrm{Au}$ collisions at $\sqrt{s_{\mathrm{NN}}}=200 \mathrm{GeV}$. The scattered dots are the experimental measurements [16-18]. The solid curves are the results from (9).

where

$$
\begin{aligned}
& S\left(\omega_{\mathrm{FO}}, y_{\mathrm{L}}\right) \\
& =\frac{\beta_{\mathrm{h}} y_{\mathrm{L}}}{\lambda\left(\omega_{\mathrm{FO}}, y_{\mathrm{L}}\right)}\left\{\frac{\beta_{\mathrm{h}} c_{\mathrm{h}} y_{\mathrm{h}}\left(\omega_{\mathrm{FO}}\right)}{\lambda\left(\omega_{\mathrm{FO}}, y_{\mathrm{L}}\right)} I_{0}\left[\lambda\left(\omega_{\mathrm{FO}}, y_{\mathrm{L}}\right)\right]\right. \\
& \left.+\left[\frac{\beta_{\mathrm{h}}+1}{\beta_{\mathrm{h}}}-\frac{2 \beta_{\mathrm{h}} c_{\mathrm{h}} y_{\mathrm{h}}\left(\omega_{\mathrm{FO}}\right)}{\lambda^{2}\left(\omega_{\mathrm{FO}}, y_{\mathrm{L}}\right)}\right] I_{1}\left[\lambda\left(\omega_{\mathrm{FO}}, y_{\mathrm{L}}\right)\right]\right\}, \\
& C\left(\omega_{\mathrm{FO}}, y_{\mathrm{L}}\right)=\left\{\frac{\beta_{\mathrm{h}}+1}{\beta_{\mathrm{h}}}+\frac{\left[\beta_{\mathrm{h}} c_{\mathrm{h}} y_{\mathrm{h}}\left(\omega_{\mathrm{FO}}\right)\right]^{2}}{\lambda^{2}\left(\omega_{\mathrm{FO}}, y_{\mathrm{L}}\right)}\right\} \\
& \cdot I_{0}\left[\lambda\left(\omega_{\mathrm{FO}}, y_{\mathrm{L}}\right)\right]+\frac{1}{\lambda\left(\omega_{\mathrm{FO}}, y_{\mathrm{L}}\right)}\left\{\frac{y_{\mathrm{h}}\left(\omega_{\mathrm{FO}}\right)}{c_{\mathrm{h}}}+1\right. \\
& \left.-\frac{2\left[\beta_{\mathrm{h}} c_{\mathrm{h}} y_{\mathrm{h}}\left(\omega_{\mathrm{FO}}\right)\right]^{2}}{\lambda^{2}\left(\omega_{\mathrm{FO}}, y_{\mathrm{L}}\right)}\right\} I_{1}\left[\lambda\left(\omega_{\mathrm{FO}}, y_{\mathrm{L}}\right)\right],
\end{aligned}
$$

where $I_{1}$ is the 1st-order modified Bessel function of the first kind.

The integral interval of $y_{\mathrm{L}}$ in $(9)$ is $\left[-y_{\mathrm{h}}, y_{\mathrm{h}}\right]$. By applying (9)-(12), together with the definitions in (7), we can get the rapidity distributions and the thermalization induced transverse momentum distributions of identified charged particles as shown in Figures 1, 2, and 3.

Figure 1 shows the rapidity distributions of identified charged particles in central Au-Au collisions at $\sqrt{s_{\mathrm{NN}}}=$ $200 \mathrm{GeV}$. The solid dots are the experimental measurements [16-18]. The solid curves are the results from (9). It can be seen that, except for proton $p$, the theoretical predictions are in good agreement with experimental data. For proton $p$, the experimental data show an evident uplift in the rapidity interval between $y=2.0$ and 3.0. This might be resulting 

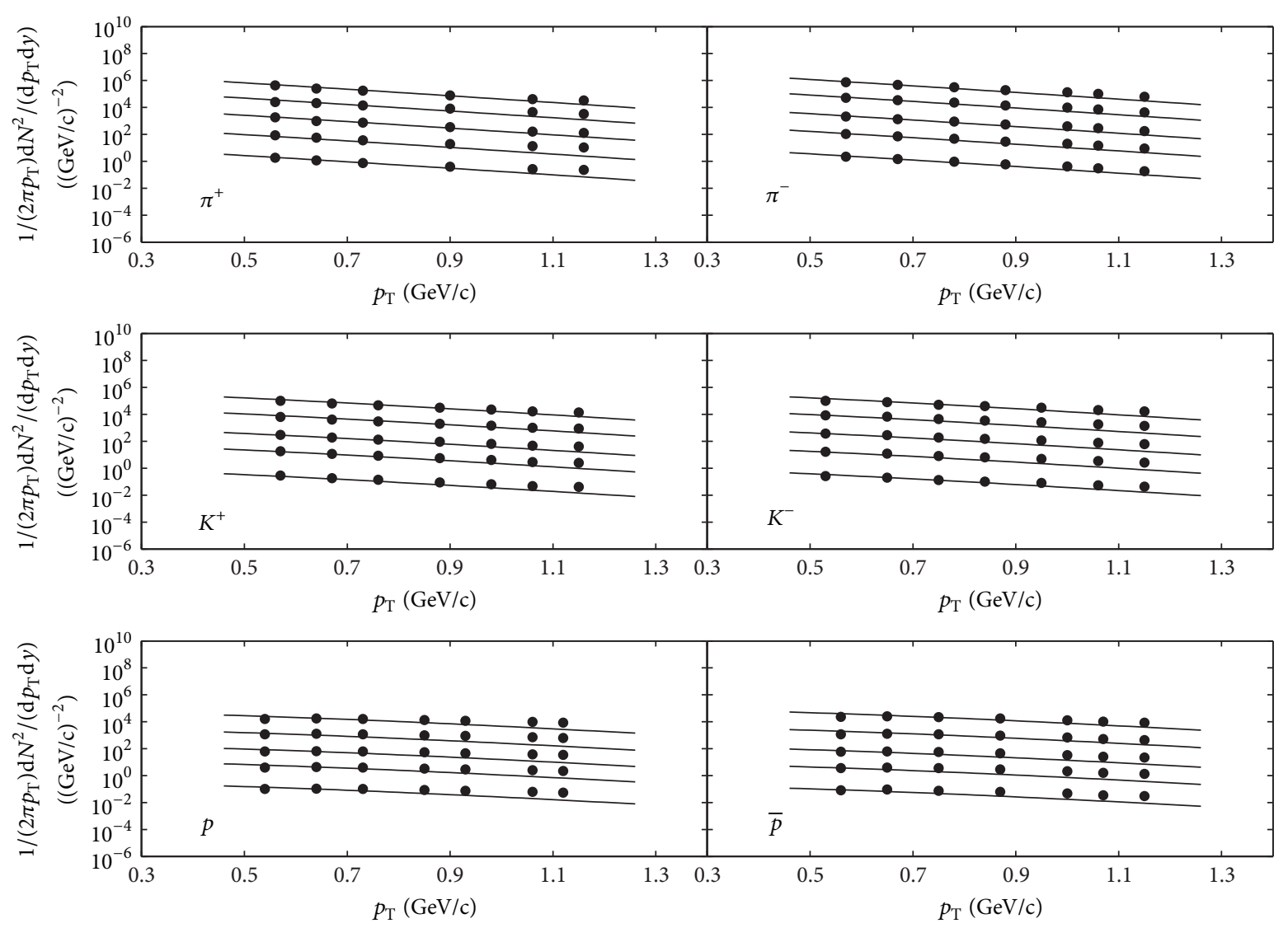

FIGURE 2: The centrality dependences of transverse momentum distributions of identified charged particles produced in Au-Au collisions at $\sqrt{s_{\mathrm{NN}}}=200 \mathrm{GeV}$. The solid dots are the experimental data [19]. The solid curves are the hydrodynamic results of (9). The centrality cuts counted from top to bottom in each panel are $0-10 \%\left(\times 10^{4}\right), 10-20 \%\left(\times 10^{3}\right), 20-40 \%\left(\times 10^{2}\right), 40-60 \%\left(\times 10^{1}\right)$, and $60-92 \%\left(\times 10^{0}\right)$, respectively.

from parts of leading particles [33, 34], which are free from the description of hydrodynamics.

In [25], by approximating the Bose-Einstein to MaxwellBoltzmann distribution for the Bose charged particles, the author also presented a rapidity distribution of $\left(\pi^{+}+\pi^{-}\right) / 2$ in Figure 2 therein, which is, however, only a theoretical prediction with no experimental comparisons.

Figures 2 and 3 are for the centrality dependences of thermalization induced transverse momentum distributions of $\pi^{ \pm}, K^{ \pm}$, and $p(\bar{p})$ in Au-Au collisions at $\sqrt{s_{\mathrm{NN}}}=200$ and $130 \mathrm{GeV}$, respectively. The left columns are for positive charged particles. The right columns are for negative ones. The centrality cuts are $0-10 \%\left(\times 10^{4}\right), 10-20 \%\left(\times 10^{3}\right)$, $20-40 \%\left(\times 10^{2}\right), 40-60 \%\left(\times 10^{1}\right)$, and $60-92 \%\left(\times 10^{0}\right)$, respectively, counted from top to bottom in Figure 2 . The centrality cuts in Figure 3 are $0-5 \%(\times 5), 5-15 \%(\times 2), 15-30 \%$, $30-60 \%$, and $60-92 \%$, respectively, counted from top to bottom. The solid dots are the experimental measurements $[19,20]$. The solid curves are the hydrodynamic predictions of (9). The corresponding values of $\chi^{2} / \mathrm{NDF}$ are listed in Tables 1 and 2 . It can be seen that the theoretical results are in good accordance with experimental data for transverse momentum up to about $p_{\mathrm{T}}=1.1 \mathrm{GeV} / \mathrm{c}$.
TABLE 1: The $\chi^{2} / \mathrm{NDF}$ and the fitted initial temperature $T_{0}$ and constant $Q_{0}$ at $68.3 \%$ confidence level in different centrality $\mathrm{Au}-\mathrm{Au}$ collisions at $\sqrt{s_{\mathrm{NN}}}=200 \mathrm{GeV}$.

\begin{tabular}{lccccc}
\hline Centrality cuts (\%) & $0-10$ & $10-20$ & $20-40$ & $40-60$ & $60-92$ \\
\hline$\chi^{2} / \mathrm{NDF}$ & & & & \\
$\pi^{+}$ & 0.92 & 1.52 & 0.75 & 2.13 & 1.44 \\
$\pi^{-}$ & 1.05 & 1.02 & 1.07 & 0.95 & 0.98 \\
$K^{+}$ & 1.02 & 0.90 & 1.48 & 1.26 & 1.30 \\
$K^{-}$ & 1.15 & 2.25 & 2.20 & 2.31 & 1.25 \\
$p$ & 3.20 & 3.64 & 3.61 & 3.26 & 3.25 \\
$\bar{p}$ & 1.34 & 1.30 & 1.65 & 2.52 & 2.33 \\
\hline$T_{0}(\mathrm{MeV})$ & $700 \pm 24$ & $618 \pm 31$ & $534 \pm 31$ & $450 \pm 35$ & $332 \pm 27$ \\
\hline \multicolumn{5}{c}{$\pi^{+} / \pi^{-}: 2.21 \pm 0.17 / 2.21 \pm 0.15 ;$} \\
$Q_{0}$ & $K^{+} / K^{-}: 1.04 \pm 0.16 / 0.98 \pm 0.19 ;$ \\
\hline \multicolumn{5}{c}{$: 2.15 \pm 0.24 / 1.61 \pm 0.20}$. \\
\hline
\end{tabular}

In the region of $p_{\mathrm{T}}>1.1 \mathrm{GeV} / \mathrm{c}$, the deviation appears as shown in Figure 4 which shows the transverse momentum distributions for $\pi^{+}$and $\pi^{-}$in Au-Au collisions at $\sqrt{s_{\mathrm{NN}}}=$ $200 \mathrm{GeV}$ for $p_{\mathrm{T}}$ up to $p_{\mathrm{T}}=2.0 \mathrm{GeV} / \mathrm{c}$. To overcome this 

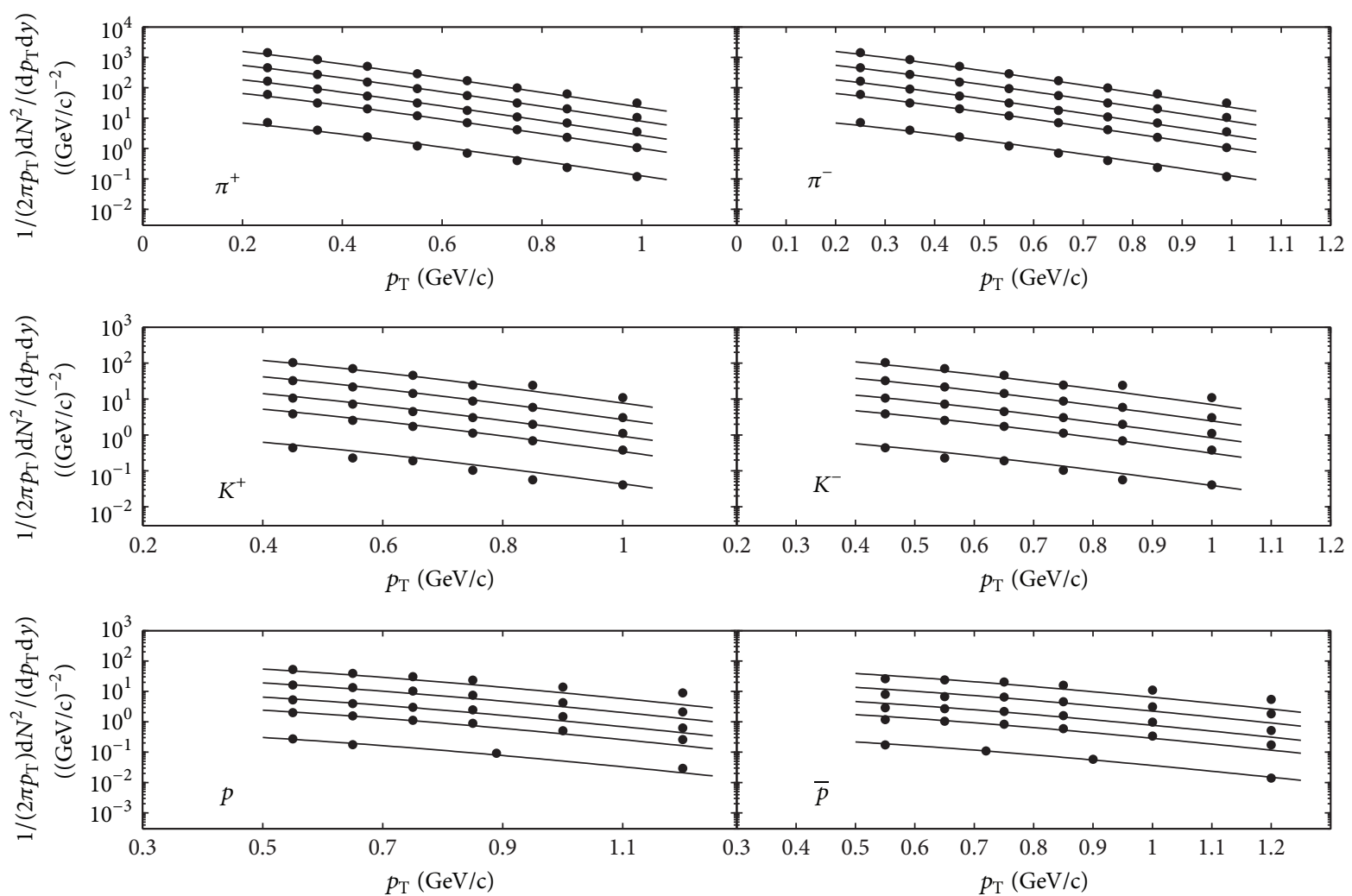

FIGURE 3: The centrality dependences of transverse momentum distributions of identified charged particles produced at midrapidity of $|y| \leq 1$ in $\mathrm{Au}-\mathrm{Au}$ collisions at $\sqrt{s_{\mathrm{NN}}}=130 \mathrm{GeV}$. The solid dots are the experimental data [20]. The solid curves are the hydrodynamic results of (9). The centrality cuts counted from top to bottom in each panel are $0-5 \%(\times 5), 5-15 \%(\times 2), 15-30 \%, 30-60 \%$, and $60-92 \%$, respectively.

TABLE 2: The $\chi^{2} / \mathrm{NDF}$ and the fitted initial temperature $T_{0}$ and constant $Q_{0}$ at $68.3 \%$ confidence level in different centrality Au-Au collisions at $\sqrt{s_{\mathrm{NN}}}=130 \mathrm{GeV}$.

\begin{tabular}{|c|c|c|c|c|c|}
\hline Centrality cuts (\%) & $0-5$ & $5-15$ & $15-30$ & $30-60$ & $60-92$ \\
\hline \multicolumn{6}{|l|}{$\chi^{2} / \mathrm{NDF}$} \\
\hline$\pi^{+}$ & 0.85 & 0.80 & 0.72 & 0.11 & 2.19 \\
\hline$\pi^{-}$ & 0.85 & 0.80 & 0.72 & 0.11 & 2.19 \\
\hline$K^{+}$ & 3.34 & 0.82 & 0.87 & 0.72 & 3.77 \\
\hline$K^{-}$ & 3.36 & 1.23 & 1.25 & 0.93 & 3.20 \\
\hline$p$ & 4.05 & 3.05 & 2.96 & 2.99 & 0.90 \\
\hline $\bar{p}$ & 4.17 & 4.02 & 3.03 & 3.02 & 0.88 \\
\hline$T_{0}(\mathrm{MeV})$ & $550 \pm 40$ & $542 \pm 36$ & $502 \pm 28$ & $403 \pm 22$ & $260 \pm 34$ \\
\hline \multirow{3}{*}{$Q_{0}$} & \multicolumn{5}{|c|}{$\pi^{+} / \pi^{-}: 3.50 \pm 0.13 / 3.50 \pm 0.13$} \\
\hline & \multicolumn{5}{|c|}{$K^{+} / K^{-}: 2.32 \pm 0.18 / 2.15 \pm 0.21$} \\
\hline & \multicolumn{5}{|c|}{$p / \bar{p}: 9.83 \pm 0.33 / 7.05 \pm 0.38$} \\
\hline
\end{tabular}

deviation, some other mechanisms should be introduced. The existing investigations have shown that if the transverse collective flow is taken into account, the describable region is extended to about $p_{\mathrm{T}}<2.0 \mathrm{GeV} / \mathrm{c}$ [20-22]. Furthermore, to describe the experimental data in the region of $p_{\mathrm{T}} \geq$ $2.0 \mathrm{GeV} / \mathrm{c}$, the contributions of blast-wave and perturbative QCD [24] should be introduced.

In calculations, $T_{c}$ in (7) takes the well-recognized value of $T_{\mathrm{c}}=180 \mathrm{MeV} \cdot c_{\mathrm{h}}$ takes the value of $c_{\mathrm{h}}=0.35$ from the investigations of $[32,47-49]$. The chemical freeze-out temperature $T_{\mathrm{FO}}$ in (9)-(12) takes $T_{\mathrm{FO}}=160 \mathrm{MeV}$ from the studies of [23], which also show that the baryochemical potential $\mu_{\mathrm{B}}$ in (9) is about equal to 20 and $27 \mathrm{MeV}$ in Au$\mathrm{Au}$ collisions at $\sqrt{s_{\mathrm{NN}}}=200$ and $130 \mathrm{GeV}$, respectively. The initial temperature $T_{0}$ in (7) takes $T_{0}=700$ and $550 \mathrm{GeV}$ in central Au-Au collisions at respective energy of $\sqrt{s_{\mathrm{NN}}}=200$ and $130 \mathrm{GeV}[32,50-53]$. This allows us to determine the constant $Q_{0}$ in (9) for different kinds of charged particles as 


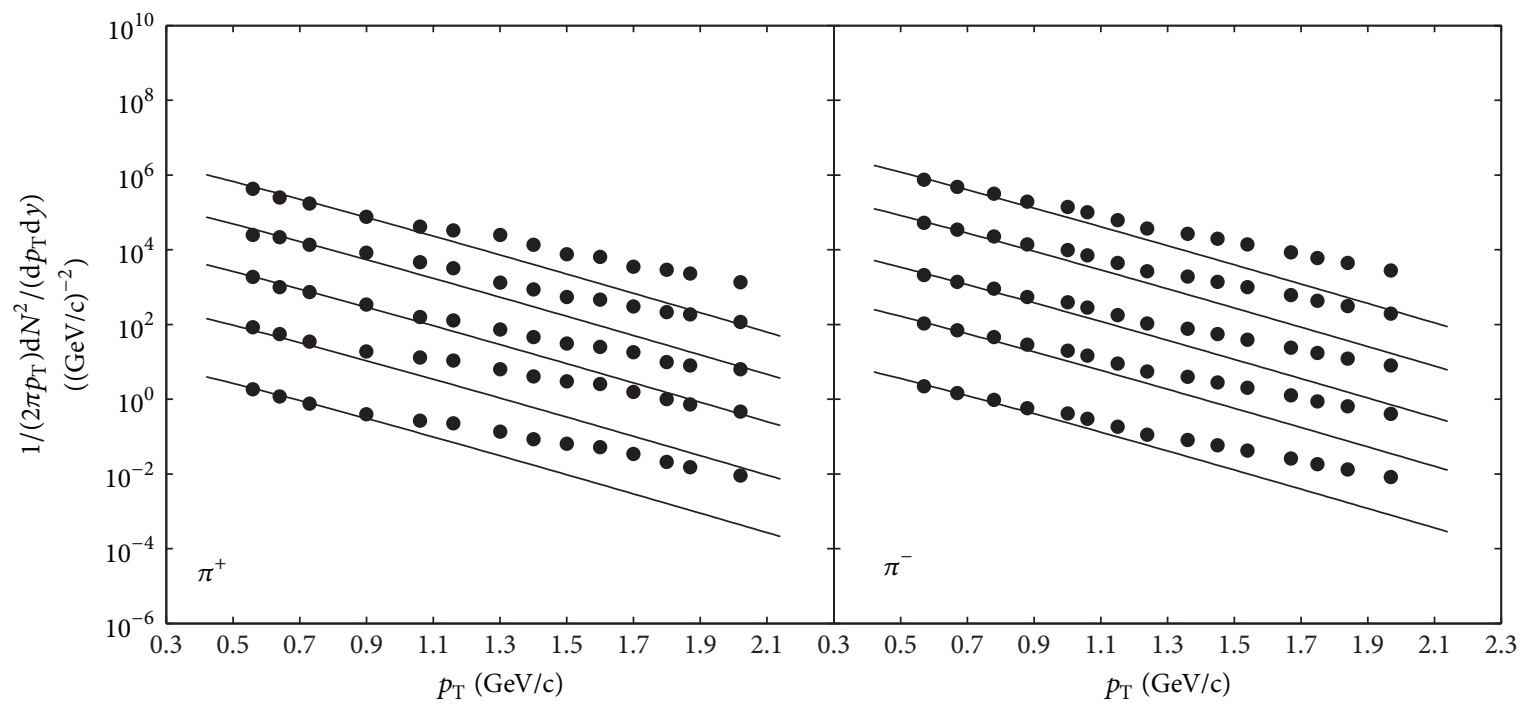

Figure 4: The transverse momentum distributions of $\pi^{+}$and $\pi^{-}$in Au-Au collisions at $\sqrt{s_{\mathrm{NN}}}=200 \mathrm{GeV}$ for $p_{\mathrm{T}}$ up to $p_{\mathrm{T}}=2.0 \mathrm{GeV} / \mathrm{c}$. The centrality cuts are the same as those in Figure 2. The solid dots are the experimental data [19]. The solid curves are the hydrodynamic results of (9).

listed in Tables 1 and 2. Keeping $Q_{0}$ unchanged, we can get $T_{0}$ in different centrality cuts as shown in Tables 1 and 2 . As we might expect, $T_{0}$ decreases with increasing centrality cuts.

The fitted results of $Q_{0}$ in Tables 1 and 2 give the ratios

$$
\begin{aligned}
\frac{Q_{0}\left(\pi^{+}\right)}{Q_{0}\left(\pi^{-}\right)} & =1, \\
\frac{Q_{0}\left(K^{+}\right)}{Q_{0}\left(K^{-}\right)} & =1.06, \\
\frac{Q_{0}(p)}{Q_{0}(\bar{p})} & =1.34
\end{aligned}
$$

$(200 \mathrm{GeV})$,

$$
\begin{aligned}
\frac{Q_{0}\left(\pi^{+}\right)}{Q_{0}\left(\pi^{-}\right)} & =1, \\
\frac{Q_{0}\left(K^{+}\right)}{Q_{0}\left(K^{-}\right)} & =1.08, \\
\frac{Q_{0}(p)}{Q_{0}(\bar{p})} & =1.39
\end{aligned}
$$

$(130 \mathrm{GeV})$.

These ratios are well consistent with the relative abundances of particles and antiparticles presented in [23]. This consistence may be attributed to the fact that, in case of adopting a common $T_{\mathrm{FO}}$ for different charged particles, the integral part of (9) is the same for particles and antiparticles. Hence, $Q_{0}$ should be proportional to the abundance of corresponding particles.

\section{Conclusions}

In order to see the importance of thermalization in the expansions of the hot and dense matter created in high energy heavy ion collisions, the transverse collective flow is as usual not taken into account. The expansion of matter in the transverse directions is therefore only induced by the thermalization of fluid. Multiplied with longitudinal collective flow, we can get the invariant multiplicity distributions of charged particles.

The model contains rich information about the transport coefficients of fluid, such as the sound speed in QGP $c_{0}$, the sound speed $c_{\mathrm{h}}$ in hadronic phase, the phase transition temperature $T_{\mathrm{c}}$, the chemical freeze-out temperature $T_{\mathrm{FO}}$, the baryochemical potential $\mu_{\mathrm{B}}$, and the initial temperature $T_{0}$. With the exception of $T_{0}$, the other five coefficients take the values either from the well-known theoretical results or from experimental measurements. As for $T_{0}$, it takes the values from other researches for the most central collisions. For the rest centrality cuts, $T_{0}$ is determined by comparing the theoretical results with experimental data.

From the coincidences between the theoretical curves and experimental data points, we can see that the theoretical model can give a good description of rapidity distributions measured in central Au-Au collisions at $\sqrt{s_{\mathrm{NN}}}=200 \mathrm{GeV}$. For the transverse momentum distributions measured in $\mathrm{Au}-\mathrm{Au}$ collisions at $\sqrt{s_{\mathrm{NN}}}=200$ and $130 \mathrm{GeV}$, the theoretical results match up well with experimental data for the transverse momentum up to about $p_{\mathrm{T}}=1.1 \mathrm{GeV} / \mathrm{c}$.

\section{Conflicts of Interest}

The authors declare that there are no conflicts of interest regarding the publication of this paper. 


\section{Acknowledgments}

This work is supported by the Shanghai Key Lab of Modern Optical System.

\section{References}

[1] E. Laermann, O. Philipsen, and Ann., "Lattice QCD at finite temperature," Annual Review of Nuclear and Particle Science, vol. 53, pp. 163-198, 2003.

[2] J. D. Bjorken, "Highly relativistic nucleus-nucleus collisions: the central rapidity region," Physical Review D, vol. 27, pp. 140-151, 1983.

[3] K. Adcox and (PHENIX Collaboration), "Measurement of the Midrapidity Transverse Energy Distribution from $\sqrt{s_{N N}} \mathrm{GeV}$ $\mathrm{Au}+\mathrm{Au}$ Collisions at RHIC," Physical Review Letters, vol. 87, Article ID 052301, 2001.

[4] I. Arsene, I. G. Bearden, D. Beavis et al., "Quark-gluon plasma and color glass condensate at RHIC? The perspective from the BRAHMS experiment," Nuclear Physics A, vol. 757, pp. 1-27, 2005.

[5] B. Back and PHOBOS Collaboration, "The PHOBOS perspective on discoveries at RHIC," Nuclear Physics A, vol. 757, pp. 28101, 2005.

[6] J. Adams and (STAR Collaboration), "Experimental and theoretical challenges in the search for the quark-gluon plasma: The STAR Collaboration's critical assessment of the evidence from RHIC collisions," Nuclear Physics A, vol. 757, pp. 102-183, 2005.

[7] K. Adcox and PHENIX Collaboration, "Formation of dense partonic matter in relativistic nucleus-nucleus collisions at RHIC: experimental evaluation by the PHENIX Collaboration," Nuclear Physics A, vol. 757, no. 1-2, pp. 184-283, 2005.

[8] M. G. Albrow, "British-French-Scandinavian Collaboration," Nucl. Phys. B, vol. 145, pp. 305-348, 1978.

[9] G. N. Fowler, E. M. Friedlander, R. M. Weiner, and G. Wilk, "Possible manifestation of quark-gluon plasma in multiplicity distributions from high-energy reactions," Physical Review Letters, vol. 57, no. 17, pp. 2119-2122, 1986.

[10] E. M. Friedlander and R. M. Weiner, "Evidence from Very Large Transverse Momenta of a Change with Temperature of Velocity of Sound in Hadronic Matter," Physical Review Letters, vol. 43, pp. 15-18, 1979.

[11] P. Lévai and B. Müller, "Transverse baryon flow as possible evidence for a quark-gluon-plasma phase," Physical Review Letters, vol. 67, no. 12, pp. 1519-1522, 1991.

[12] T. Alexopoulos, "Mass-identified particle production in protonantiproton collisions at $\sqrt{s}=300,540,1000$, and $1800 \mathrm{GeV}$," Physical Review D, vol. 48, pp. 984-997, 1993.

[13] L. Adamczyk and STAR Collaboration, "Centrality dependence of identified particle elliptic flow in relativistic heavy ion collisions at $\sqrt{s_{N N}}=7.7-62.4 \mathrm{GeV}$,' Physical Review C, vol. 93, Article ID 014907, 2016.

[14] J. Adam and ALICE Collaboration, "Anisotropic flow of charged particles in $\mathrm{Pb}-\mathrm{Pb}$ collisions at $\sqrt{s_{N N}}=5.02 \mathrm{TeV}$," Physical Review Letters, vol. 116, Article ID 132302, 2016.

[15] B. Alver and PHOBOS Collaboration, "Charged-particle multiplicity and pseudorapidity distributions measured with the PHOBOS detector in $\mathrm{Au}+\mathrm{Au}, \mathrm{Cu}+\mathrm{Cu}, \mathrm{d}+\mathrm{Au}$, and $\mathrm{p}+\mathrm{p}$ collisions at ultrarelativistic energies," Physical Review C, vol. 83, Article ID 024913, 2011.
[16] M. Murray (for the BRAHMS Collaboration), "Scanning the phases of QCD with BRAHMS," Journal of Physics G: Nuclear and Particle Physics, vol. 30, p. 667, 2004.

[17] M. Murray (for the BRAHMS Collaboration), "Flavor dynamics," Journal of Physics G: Nuclear and Particle Physics, vol. 35, Article ID 044015, 2008.

[18] I. G. Bearden and BRAHMS Collaboration, "Charged meson rapidity distributions in central $\mathrm{Au}+\mathrm{Au}$ collisions at $\sqrt{s_{N N}}=$ 200 GeV," Physical Review Letters, vol. 94, Article ID 162301, 2005.

[19] A. Adare and PHENIX Collaboration, "Spectra and ratios of identified particles in $\mathrm{Au}+\mathrm{Au}$ and $\mathrm{d}+\mathrm{Au}$ collisions at $\sqrt{s_{N N}}=$ 200 GeV," Physical Review C, vol. 88, Article ID 024906, 2013.

[20] K. Adcox and PHENIX Collaboration, "Single identified hadron spectra from $\sqrt{s_{N N}}=130 \mathrm{GeV} \mathrm{Au}+\mathrm{Au}$ collisions," Physical Review C, vol. 69, Article ID 024904, 2004.

[21] C. Adler and STAR Collaboration, "Measurement of inclusive antiprotons from $\mathrm{Au}+\mathrm{Au}$ collisions at $\sqrt{s_{N N}}=130 \mathrm{GeV}$," Physical Review Letters, vol. 87, Article ID 262302, 2001.

[22] K. Adcox and PHENIX Collaboration, "Centrality dependence of $\pi^{+/-}, K^{+/-}, p$, and $\bar{p}$ production from $\sqrt{s_{N N}}=130 \mathrm{GeV} \mathrm{Au}+$ Au collisions at RHIC," Physical Review Letters, vol. 88, Article ID 242301, 2002.

[23] B. I. Abelev and STAR Collaboration, "Systematic measurements of identified particle spectra in pp, $d+A u$, and $A u+A u$ collisions at the STAR detector," Physical Review C, vol. 79, Article ID 034909, 2009.

[24] B. Abelev and ALICE Collaboration, "Centrality dependence of $\pi, K$, and $p$ production in $\mathrm{Pb}-\mathrm{Pb}$ collisions at $\sqrt{s_{N N}}=2.76 \mathrm{TeV}$," Physical Review C, vol. 88, Article ID 044910, 2013.

[25] N. Suzuki, "One-dimensional hydrodynamical model including phase transition," Physical Review C, vol. 81, Article ID 044911, 2010.

[26] T. S. Biró, "Generating new solutions for relativistic transverse flow at the softest point," Physics Letters B, vol. 487, pp. 133-139, 2000.

[27] T. S. Biró, "Analytic solution for relativistic transverse flow at the softest point," Physics Letters. B. Particle Physics, Nuclear Physics and Cosmology, vol. 474, no. 1-2, pp. 21-26, 2000.

[28] M. Csanád, M. I. Nagy, and S. Lökös, "Exact solutions of relativistic perfect fluid hydrodynamics for a QCD Equation of State," The European Physical Journal A, vol. 48, pp. 173-178, 2012.

[29] T. Csörgo and G. Kasza, arXiv:1610.0219 /nucl-th (2016).

[30] T. Csörgo, M. I. Nagy, and M. Csanád, "New family of simple solutions of relativistic perfect fluid hydrodynamics," Physics Letters B, vol. 663, pp. 306-311, 2008.

[31] F. Cooper and G. Frye, "Landau's hydrodynamic model of particle production and electron-positron annihilation into hadrons," Physical Review D, vol. 11, pp. 192-213, 1975.

[32] T. Mizoguchi, H. Miyazawa, and M. Biyajima, "A potential including the Heaviside function in the $1+1$ dimensional hydrodynamics by Landau: IIts basic properties and application to data at RHIC energies," European Physical Journal A, vol. 40, no. 1, pp. 99-108, 2009.

[33] Z. J. Jiang, Q. G. Li, and H. L. Zhang, "Revised Landau hydrodynamic model and the pseudorapidity distributions of charged particles produced in nucleus-nucleus collisions at maximum energy at the BNL Relativistic Heavy Ion Collider," Physical Review C, vol. 87, Article ID 044902, 2013. 
[34] Z. J. Jiang, Y. Zhang, H. L. Zhang, and H. P. Deng, "A description of the pseudorapidity distributions in heavy ion collisions at RHIC and LHC energies," Nuclear Physics A, vol. 941, pp. 188200, 2015.

[35] E. K. G. Sarkisyan, A. N. Mishra, R. Sahoo, and A. S. Sakharov, "Erratum: Multihadron production dynamics exploring the energy balance in hadronic and nuclear collisions (Physical Review D - Particles, Fields, Gravitation and Cosmology (2016) D93 (054046))," Physical Review D - Particles, Fields, Gravitation and Cosmology, vol. 93, no. 7, Article ID 079904, 2016.

[36] E. K. G. Sarkisyan, A. N. Mishra, R. Sahoo, and A. S. Sakharov, "Centrality dependence of midrapidity density from $\mathrm{GeV}$ to $\mathrm{TeV}$ heavy-ion collisions in the effective-energy universality picture of hadroproduction," Physical Review D, vol. 94, Article ID 011501, 2016.

[37] C. Gale, S. Jeon, and B. Schenke, "Hydrodynamic modeling of heavy-ion collisions," International Journal of Modern Physics A, vol. 28, Article ID 1340011, 2013.

[38] U. Heinz and R. Snellings, "Collective flow and viscosity in relativistic heavy-ion collisions," Annual Review of Nuclear and Particle Science, vol. 63, pp. 123-151, 2013.

[39] A. Bialas and R. Peschanski, "Asymmetric $(1+1)$-dimensional hydrodynamics in high-energy collisions," Physical Review C, vol. 83, Article ID 054905, 2011.

[40] A. Bialas, R. A. Janik, and R. Peschanski, "Unified description of Bjorken and Landau $1+1$ hydrodynamics," Physical Review C, vol. 76, Article ID 054901, 2007.

[41] G. Beuf, R. Peschanski, and E. N. Saridakis, "Entropy flow of a perfect fluid in $(1+1)$ hydrodynamics," Physical Review C, vol. 78, no. 6, Article ID 064909, 2008.

[42] C. Y. Wong, "Landau hydrodynamics reexamined," Physical Review C, vol. 78, Article ID 054902, 2008.

[43] E. K. G. Sarkisyan and A. S. Sakharov, "Relating multihadron production in hadronic and nuclear collisions," European Physical Journal C, vol. 70, no. 3, pp. 533-541, 2010.

[44] A. N. Mishra, R. Sahoo, E. K. G. Sarkisyan, and A. S. Sakharov, "Effective-energy budget in multiparticle production in nuclear collisions," The European Physical Journal C, vol. 74, p. 3147, 2014.

[45] J. Noronha-Hostler, M. Luzum, and J.-Y. Ollitrault, "Hydrodynamic predictions for $5.02 \mathrm{TeV} \mathrm{Pb}-\mathrm{Pb}$ collisions," Physical Review C-Nuclear Physics, vol. 93, no. 3, Article ID 034912, 2016.

[46] H. Niemi, K. J. Eskola, and R. Paatelainen, "Event-by-event fluctuations in a perturbative QCD + saturation + hydrodynamics model: Determining QCD matter shear viscosity in ultrarelativistic heavy-ion collisions," Physical Review C, vol. 93, Article ID 024907, 2016.

[47] A. Adare and PHENIX Collaboration, "Scaling properties of azimuthal anisotropy in $\mathrm{Au}+\mathrm{Au}$ and $\mathrm{Cu}+\mathrm{Cu}$ collisions at $\sqrt{{s_{N N}}}$ $=200$ GeV," Physical Review Letters, vol. 98, Article ID 162301, 2007.

[48] L. N. Gao, Y. H. Chen, H. R. Wei, and F. H. Liu, "Speed of sound parameter from RHIC and LHC heavy-ion data," Advances in High Energy Physics, vol. 2014, Article ID 450247, 8 pages, 2014.

[49] S. Borsányi, G. Endrodi, Z. Fodor et al., "The QCD equation of state with dynamical quarks," Journal of High Energy Physics, vol. 2010, no. 11, article 77, pp. 1-31, 2010.

[50] S. turbide, R. Rapp, and C. Gale, "Hadronic production of thermal photons," Physical Review C, vol. 69, Article ID 014903, 2004.

[51] A. Adare and PHENIX Collaboration, "Enhanced production of direct photons in $\mathrm{Au}+\mathrm{Au}$ collisions at $\sqrt{s_{N N}}=200 \mathrm{GeV}$ and implications for the initial temperature," Physical Review Letters, vol. 104, Article ID 132301, 2010.

[52] D. D'Enterria and D. Peressounko, "Probing the QCD equation of state with thermal photons in nucleus-nucleus collisions at RHIC," European Physical Journal C, vol. 46, no. 2, pp. 451-464, 2006.

[53] M. Csanád and I. Májer, "Equation of state and initial temperature of quark gluon plasma at RHIC," Central European Journal of Physics, vol. 10, no. 4, pp. 850-857, 2012. 

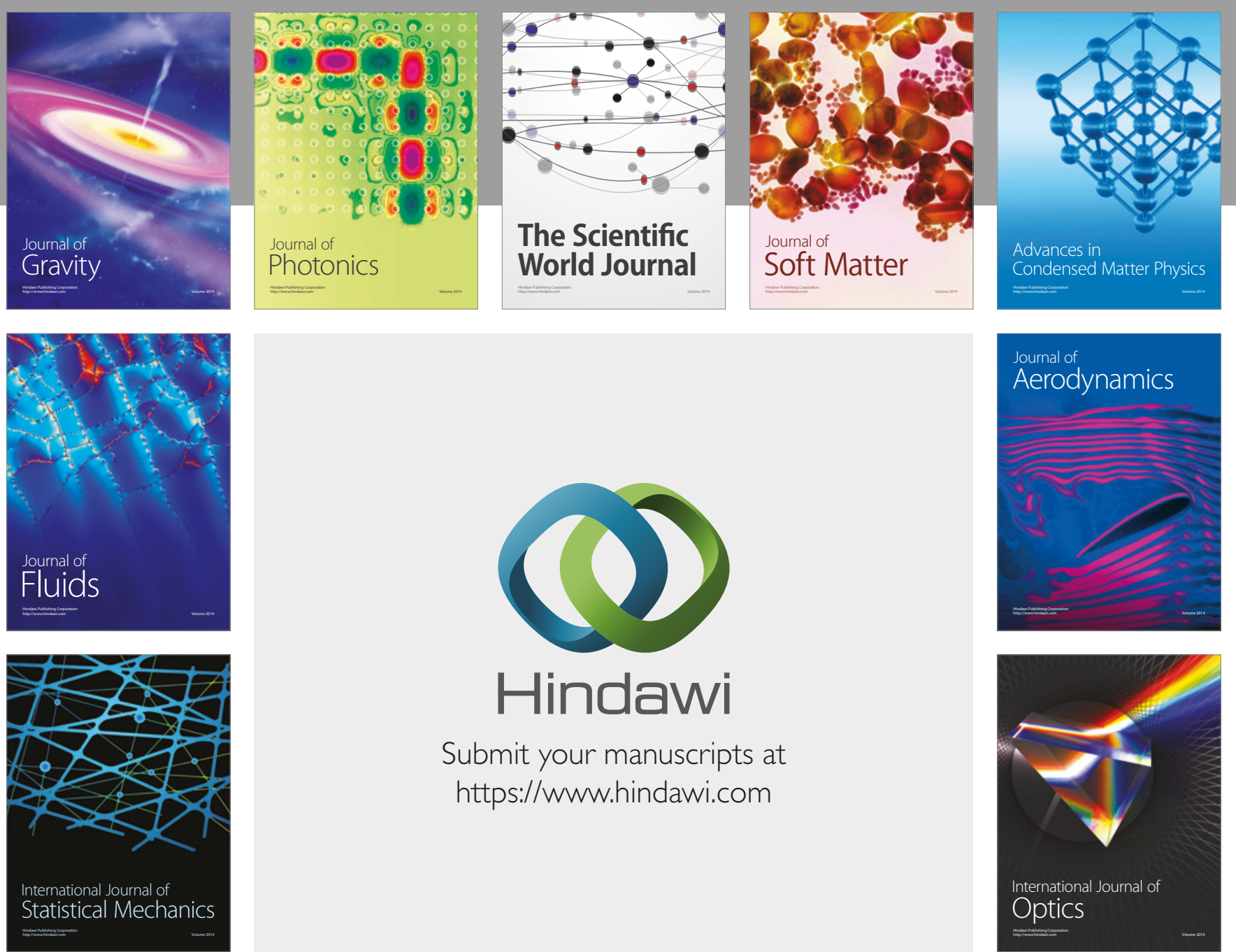

Submit your manuscripts at

https://www.hindawi.com
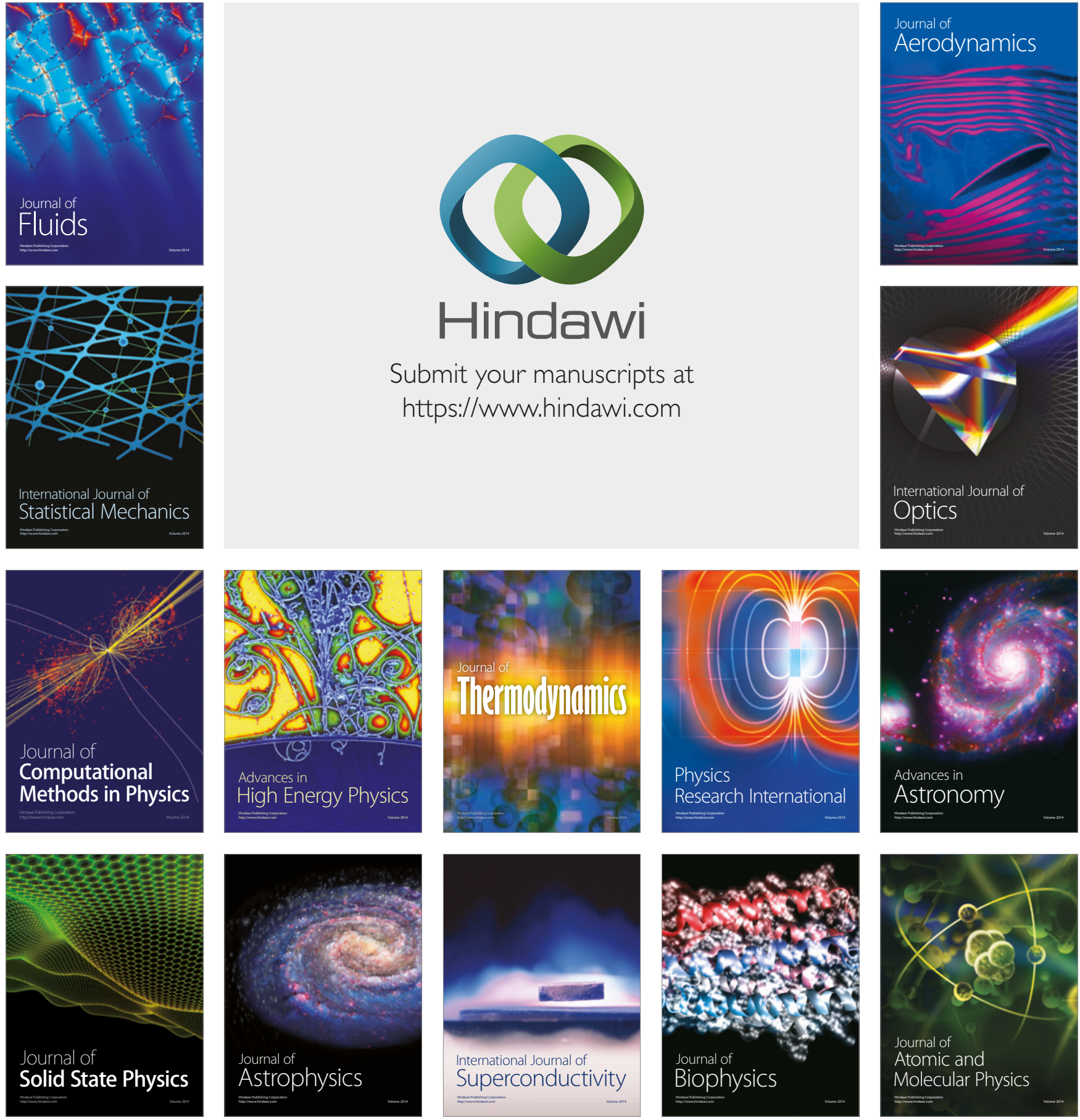